\title{
Temperature Dependence of the Flory Interaction Parameter in a Single-Phase Mixture of Poly(hydroxy ether of bisphenol-A) and Poly(ether sulfone)
}

\author{
Hiromu SAITO, Daisuke TsuTSUmI, ${ }^{\dagger}$ and Takashi INOUE* \\ Department of Organic and Polymeric Materials, \\ Tokyo Institute of Technology, Ookayama, \\ Meguro-ku, Tokyo 152, Japan
}

(Received April 24, 1989)

\begin{abstract}
The Flory interaction parameter $\chi_{12}$ in a single-phase mixture of poly(ether sulfone) and poly(hydroxy ether of bisphenol-A) was measured at various temperatures by the light scattering method based on random phase approximation. The $\chi_{12}$ value at two-phase region above LCST (lower critical solution temperature) was estimated by curve fitting of the Flory-Huggins theory to the binodal curve observed. Combining the results, temperature dependence of $\chi_{12}$ was obtained in a wide range. The interactional energy contribution in $\chi_{12}, \chi_{\mathrm{H}}$, was estimated by the infrared spectroscopy method based on the acid-base interaction theory by Drago. The difference between $\chi_{12}$ and $\chi_{\mathrm{H}}$, which is assumed to be the free volume term in $\chi_{12}$, was found to be a concave function of temperature; i.e., $\chi(T)$ is an increasing function at high temperature region, as has been predicted theoretically, but it is a decreasing function at low temperature region. This may lead to a thermodynamic interpretation of the appearance of both LCST and UCST (upper critical solution temperature) in mixtures of dissimilar polymers with high molecular weights, as reported in recent literature.
\end{abstract}

KEY WORDS Polymer Blend / Poly(ether sulfone) / Poly(hydroxy ether of Bisphenol-A) / Light Scattering / FT-IR / Free Volume Term / Acid-Base Interaction /

It is well known that the miscibility of dissimilar polymers with high molecular weights is mostly attained by the negative contribution of the Flory interaction parameter $\chi_{12}$ to the free energy of mixing, since the combinatorial entropy of mixing is very small. On the basis of recent theories on polymer-polymer miscibility, ${ }^{1} \chi_{12}$ is assumed to consist of the interactional energy term $\chi_{\mathbf{H}}$ and "free volume" term $\chi_{\mathrm{F}}$ arising from mismatch of the equation-of-state parameters. Miscible polymers tend to phase separate at elevated temperatures. This lower critical solution temperature (LCST) behavior is interpreted by the crossover of increasing positive $\chi_{\mathrm{F}}$ and nega- tive $\chi_{\mathbf{H}}$ with elevating temperature. Recently, some miscible polymers were found to phaseseparate also at lower temperature, namely, they exhibit upper critical solution temperature (UCST) behavior. ${ }^{2-4}$ The coexistence of LCST and UCST behavior can be interpreted presumably by the concave character of the temperature dependence of $\chi_{\mathrm{F}}{ }^{5,6}$ Thus the empirical justification of the temperature dependence of $\chi_{\mathrm{F}}$ is an interesting subject in the polymer blends.

There are various methods to estimate the $\chi_{12}$ value; e.g., melting point depression, ${ }^{7}$ inverse-phase gas chromatography, ${ }^{8}$ and small angle $\mathrm{X}$-ray and neutron scattering

${ }^{\dagger}$ On leave from Research and Development Department, Toyoda Gosei Co., Ltd., Kitajima, Inazawa 492, Japan.

* To whom correspondence should be addressed. 
methods. ${ }^{5,9,10}$ To estimate $\chi$ values at various temperatures, we prefer the scattering method based on the random phase approximation. ${ }^{11}$ Recent infrared spectroscopic analysis has made it possible to estimate $\chi_{\mathrm{H}}$. It was obtained from the extensive studies by Drago $^{12}$ on the acidity and basicity of small molecules for which frequency shift-enthalpy correlations have been established. Fowkes et al. ${ }^{13}$ and Kwei et al. ${ }^{14}$ extended the IR method to polymer systems and established similar correlations. We apply this method to estimate the temperature dependence of $\chi_{H}$ in a polymer-polymer mixture.

In this paper, the poly(hydroxy ether of bisphenol-A) (Phenoxy)/poly(ether sulfone) (PES) system is used because (1) this system is known to exhibit LCST at around $200^{\circ} \mathrm{C}^{15}$ which provides favorable conditions for light scattering experiments on a singlephase mixture, and (2) Phenoxy possesses hydroxyl group, a sensitive moiety to the frequency shift with the interaction. ${ }^{16} \mathrm{We}$ investigate the temperature dependence of $\chi_{12}$ and $\chi_{\mathrm{H}}$. at the single phase region. We also observe the binodal curve by the cloud point method. By the curve fitting of the FloryHuggins theory to the observed binodal curve, we obtain the temperature dependence of $\chi_{12}$ at two-phase region. Then we discuss the temperature dependence of $\chi_{\mathrm{F}}$, which is assumed to be the difference between $\chi_{12}$ and $\chi_{\mathrm{H}}$.

\section{EXPERIMENTAL}

The polymer specimens used in this study were commercial polymers. Phenoxy was supplied by Union Carbide Co., Ltd. (PKHH, $M_{n}=2.5 \times 10^{4}$ ). PES was supplied by Sumitomo Chemical Co., Ltd. (4100G, $M_{n}=1.8 \times$ $\left.10^{4}\right)$.

Phenoxy and PES were dissolved at $10 \mathrm{wt} \%$ of total polymer in dimethyl formamide (DMF). The solution was cast onto a cover glass after filtration through a micropore filter having a pore size $0.45 \mu \mathrm{m}$. The solvent was evaporated under reduced atmosphere of $10^{-2} \mathrm{mmHg}$ at $40^{\circ} \mathrm{C}$. The transparent cast film was dried under vacuum $\left(10^{-4} \mathrm{mmHg}\right)$ at $70^{\circ} \mathrm{C}$ for 3 days, and further at $130^{\circ} \mathrm{C}$ for $24 \mathrm{~h}$. The thick film thus prepared ( $c a .60 \mu \mathrm{m}$ thick) was used for the cloud point measurement and the light scattering experiment. A thin film (ca. $10 \mu \mathrm{m}$ thick) was also prepared for infrared spectroscopy by casting a $3 \mathrm{wt} \%$ DMF solution onto $\mathrm{KBr}$ disk and dried in the same way as mentioned above. In order to obtain the enthalpy-infrared frequency shift correlation for our acidic polymer, a Phenoxy solution in benzene ( $c a .0 .02 \mathrm{moll}^{-1}$ ) was prepared. The solution was mixed with a series of small molecule bases: 1,4-dioxane, tetrahydrofuran, $N, N$-dimethylacetamide and pyridine (ca. $0.04 \mathrm{moll}^{-1}$ level). The mixed solution was filled in a KSR liquid cell with optical path of $1 \mathrm{~mm}$.

A phase diagram of the Phenoxy/PES mixture was obtained by the cloud point method. The transparent film specimen was isothermally annealed at various temperatures for $72 \mathrm{~h}$. When the specimen became opaque and two-phase morphology was observed under an optical microscope, we judged that the blend was annealed in the two-phase region in the phase diagram. When no appreciable change with annealing was detected, we judged that the blend was in the single-phase region.

A He-Ne gas laser beam of $632.8 \mathrm{~nm}$ wavelength was applied vertically to the film specimen. The specimen was put into a thermoregurated silicone oil bath in quartz cell. The measurement was carried out after isothermal annealing for $2 \mathrm{~h}$. The angular distribution of $V_{\mathrm{v}}$ (parallely polarized) light scattering intensity was detected by a photomultiplier (R268, Hamamatsu Photonics Co., Ltd.) mounted on a goniometer. The scattering intensity $I(q)$ was given after the correction ${ }^{17}$ :

$$
\begin{gathered}
I(q)=C_{n} \cdot I_{0}(q) \\
C_{\mathrm{n}}=n^{2}\left[1-(\sin \theta / n)^{2}\right]^{1 / 2} / \cos \theta
\end{gathered}
$$


where $I_{0}(q)$ is the observed scattering intensity at the scattering angle $\theta, q$ is the magnitude of the scattering wave vector given by $q=(4 \pi /$ 2) $\sin (\theta / 2) ; \lambda$ being the wavelength of the light in the specimen, $n$ is the refractive index of the film specimen. The measurement was carried out after isothermal annealing for $2 \mathrm{~h}$.

Fourier transform infrared spectroscopy (FT-IR) was carried out by FT-IR/5000 spectrometer with $4 \mathrm{~cm}^{-1}$ resolution (Japan Spectroscopic Co., Ltd.). A high temperature cell was used to obtain the spectra at high temperatures. Spectra at high temperatures were measured after holding for at least $2 \mathrm{~h}$.

\section{RESULTS AND DISCUSSION}

\section{Phase Behavior}

All the solution cast films of various compositions were optically clear. The clarity was maintained even after annealing for $72 \mathrm{~h}$ at low temperatures. This behavior is indicated by the open circles in Figure 1. After the annealing, some films became translucent and a twophase structure with weak contrast was observed under a microscope. This is indicated by the triangles in Figure 1. The crosses in Figure 1 represent the situation where the film became opaque and an irregular two-phase structure definitely developed after annealing. On the basis of these observations, the LCST line was

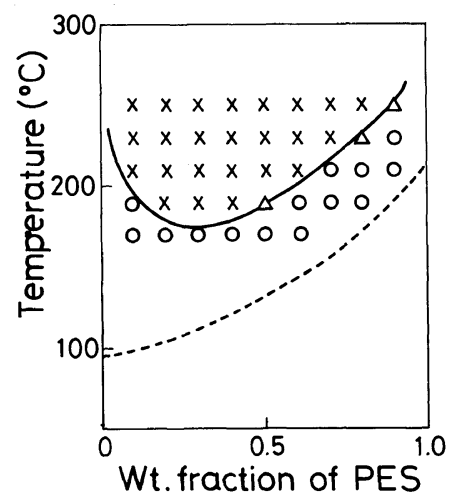

Figure 1. Phase diagram of Phenoxy/PES. Broken line is the glass transition temperature calculated by the Fox equation. drawn somewhat arbitrary in Figure 1.

Once the phase diagram is obtained, one can estimate the temperature dependence of $\chi_{12}$ by assuming $^{18}$

$$
\chi_{12}=A / T+B T+C
$$

where $T$ is temperature and $A, B$, and $C$ are constants. On the basis of the Flory-Huggins equation, one can calculate the binodal curve for a given set of parameters $A, B$, and $C$ in eq 3 , the molecular weights of component polymers, and the critical temperature by light scattering analysis (Figure 3). We selected the best set of parameter $C$ to fit the calculated binodal curve to the phase diagram observed. The results are shown by the $\chi_{12}-T$ curve in Figure 8 (broken line above LCST).

\section{Temperature Dependence of $\chi_{12}$}

The light scattering intensity I of a critical mixture, 70/30 Phenoxy/PES, decreased monotonously with increasing magnitude of the scattering wave vector $q$ at various temperatures in single-phase region. The results are shown by the $I^{-1}$ versus $q^{2}$ plots in Figure 2 . The scattering intensity increases with temperature up to LCST. This can be ascribed to the concentration fluctuation in homogeneous single-phase mixture and the scattering

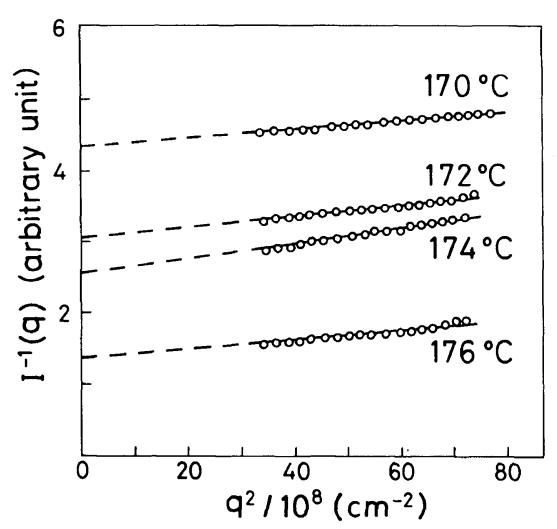

Figure 2. Reciprocal of light scattering intensity $I^{-1}$ as a function of the square of the magnitude of scattering wave vector $q^{2}$ at various temperatures in single-phase region, 70/30 Phenoxy/PES (critical mixture). 


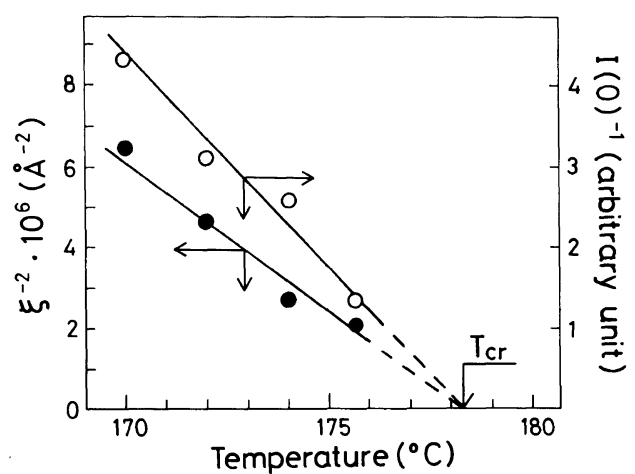

Figure 3. Temperature dependence of $I(0)^{-1}$ and $\xi^{-2}$.

is described in terms of the de Gennes scattering function $S(q)$ from the mean-field random phase approximation (RPA), ${ }^{13}$

$$
\begin{gathered}
\frac{1}{S(q)}=\frac{I(q) / I(0)=S(q) / S(0)}{N_{1} \phi_{1} g_{\mathrm{D}}\left(R_{\mathrm{g} 1}{ }^{2} q^{2}\right)} \\
+\frac{1}{N_{2} \phi_{2} g_{\mathrm{D}}\left(R_{\mathrm{g} 2}{ }^{2} q^{2}\right)}-2 \chi_{12} \\
g_{\mathrm{D}}(x)=2 x^{-2}\left(x-1+\mathrm{e}^{-x}\right)
\end{gathered}
$$

where $N$ is the number of segments per polymer chain, $\phi$ is the volume fraction, $g_{\mathrm{D}}(x)$ is the Debye function for the Gaussian chain and $R_{\mathrm{g}}$ is the radius of gyration of chain (in the Gaussian chain, $R_{\mathrm{g}}{ }^{2}=N a^{2} / 6$; $a$ being the Kuhn segment length). The plot of $I(q)^{-1}$ versus $q^{2}$ at small $q$ regime $\left(5.8 \times 10^{4} \leqq q \leqq 8.4 \times 10^{4} \mathrm{~cm}^{-1}\right)$ yielded a straight line as expected from the familiar Ornstein-Zernike form

$$
I(0) / I(q)=1+\xi^{2} q^{2}
$$

which is derived from the $S(q)$ by assuming $R_{\mathrm{g}} q \ll 1$. $\xi$ in eq 7 is the correlation length of concentration fluctuation defined by

$$
\xi^{2}=\frac{R_{\mathrm{g}}{ }^{2}}{3}\left|\frac{\chi_{12}}{\chi_{\mathrm{s}}-\chi_{12}}\right|
$$

where $\chi_{\mathrm{s}}$ is the value of $\chi_{12}$ at the spinodal point given by

$$
2 \chi_{\mathrm{s}}=1 /\left(N_{1} \phi_{1}\right)+1 /\left(N_{2} \phi_{2}\right)
$$

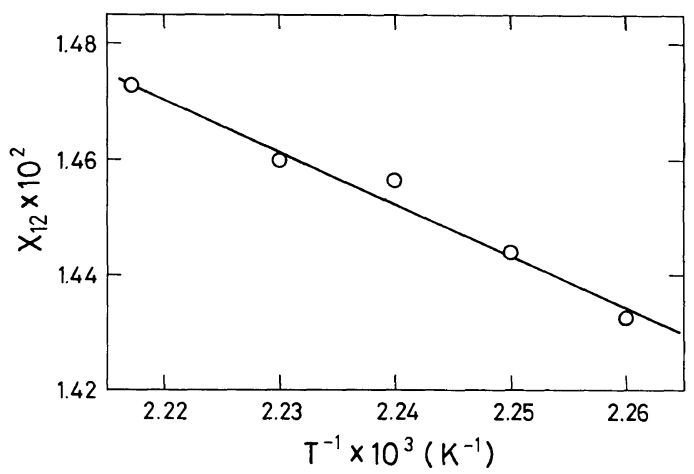

Figure 4. Temperature dependence of $\chi_{12}$, by light scattering.

The $I(0)^{-1}$ and $\xi^{-2}$, given by the intercept and slope of the plots in Figure 2; respectively, are shown as a function of temperature in Figure 4. $\xi$ increases with temperature and then diverses at a critical temperature $T_{\mathrm{cr}}\left(=178.2^{\circ} \mathrm{C}\right)$. The $T_{\text {cr }}$ derived from $\xi^{-2} \rightarrow 0$ is equal to that from $I(0)^{-1} \rightarrow 0$, suggesting the validity of RPA analysis. As aforementioned, $T_{\mathrm{cr}}$ was used to estimate the $\chi_{12}(T)$ curve by the curve fitting procedure.

Assuming $R_{\mathrm{g}}=8 \mathrm{~nm}$, the $\chi_{12}$ values were estimated by eq 8 and 9 . The results are shown as a function of temperature in Figure 4. The temperature dependence is best fitted by

$$
\chi_{12}(T)=-6.7 / T+0.043
$$

\section{Temperature Dependence of $\chi_{\mathrm{H}}$}

The whole FT-IR spectrum of the critical mixture did not show dramatic deviation from the additive spectrum of the component polymers. However, a small but distinct deviation was observed in the $\mathrm{O}-\mathrm{H}$ stretching frequency region around $3500 \mathrm{~cm}^{-1}$. This is why the spectrum is presented in a limited range of $3000-4000 \mathrm{~cm}^{-1}$ in Figure 5. At high temperature, peak position is at high frequency and a shoulder appears. The shoulder may indicate specific interactions. According to Coleman et al. ${ }^{16}$ the $\mathrm{OH}$ band in Phenoxy/ poly( $\varepsilon$-caprolactone) mixture consists of three components, "free (unassociated)" $\mathrm{OH}$, "in- 


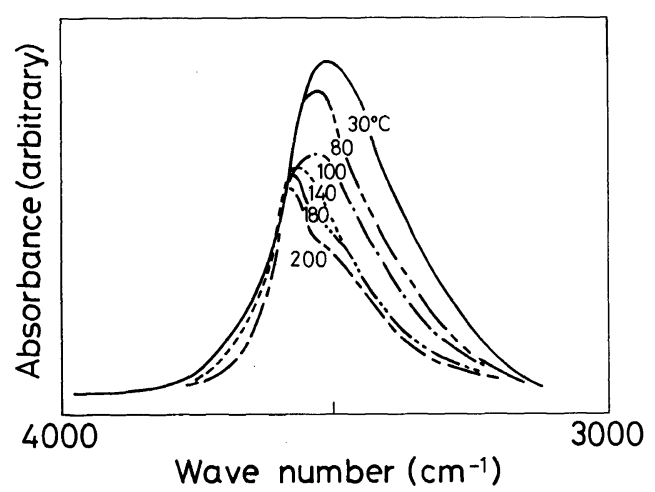

Figure 5. FT-IR spectra covering the hydroxyl stretching frequency range.

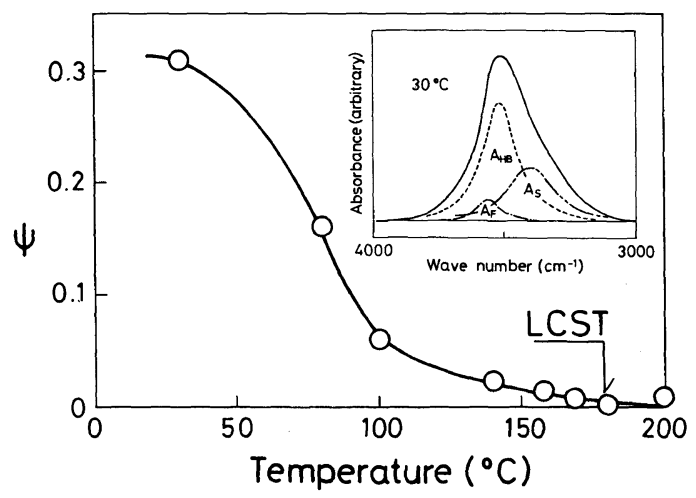

Figure 6. Mole fraction of the hydrogen-bonded $\mathrm{OH}$; $\psi=A_{\mathrm{HB}} /\left(A_{\mathrm{F}}+A_{\mathrm{S}}+A_{\mathrm{HB}}\right)$, as a function of temperature.

termolecular hydrogen-bonded" $\mathrm{OH}$, and "self-associated" $\mathrm{OH}$. The observed spectra were successfully resolved into three Lorentzian curves, as shown in the inset of Figure 6. A resolved band at intermediate frequency decreased sharply with elevating temperature, while that of high frequency increased sharply. Hence, we assigned the resolved band at $3560 \mathrm{~cm}^{-1}$ to the free $\mathrm{OH}$, at $3520 \mathrm{~cm}^{-1}$ to the hydrogen-bonded $\mathrm{OH}$, and at $3400 \mathrm{~cm}^{-1}$ to self-associated $\mathrm{OH}$, respectively. The mole fraction $\psi$ of the hydrogenbonded $\mathrm{OH}$ was assumed to be estimated by the ratio of peak areas $^{19}: \psi=A_{\mathrm{HB}} /\left(A_{\mathrm{F}}+\right.$ $A_{\mathrm{HB}}+A_{\mathrm{S}}$ ) (see, inset of Figure 6). The temperature dependence of $\psi$ is shown in Figure 6. This indicates that the number of hydrogen-

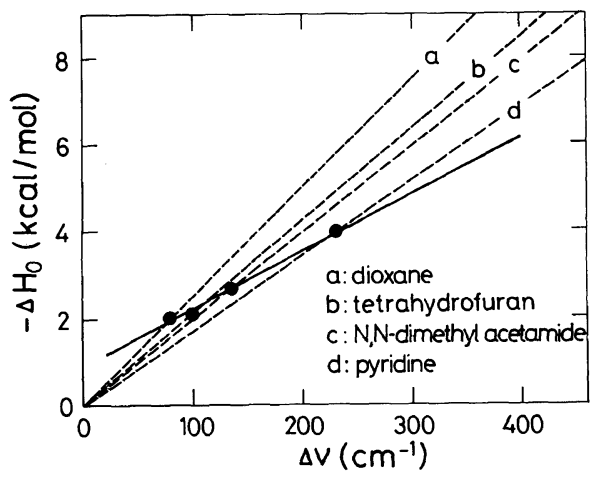

Figure 7. IR frequency shift-enthalpy correlation. Broken lines are for small molecule acids and bases (reproduced from ref 14). Closed circles are $\Delta v$ plots of the $\mathrm{OH}$ band in a dilute solution of Phenoxy in low molecule bases $(\mathrm{a}-\mathrm{d})$.

bonded $\mathrm{OH}$ decreases with temperature below LCST.

The shift of peak position of the hydrogenbonded $\mathrm{OH}$ from the free $\mathrm{OH}$ position, $\Delta v$, is a measure of the strength of intermolecular hydrogen-bonding. As described above, the hydrogen-bonded $\mathrm{OH}$ peak was resolved to appear at $3520 \mathrm{~cm}^{-1}$ and the shift with temperature was hardly detected in the PES/ Phenoxy mixture.

The correlation between the $\Delta v$ and the enthalpy of hydrogen-bonding formation $\Delta H_{0}$ for a series of small molecule bases with small molecule acids has been established by Drago et al. ${ }^{12}$ Some results for the bases in the present study are reproduced by broken lines in Figure 7. The frequency shifts of the $\mathrm{OH}$ band of Phenoxy (acid polymer) observed in the mixtures with the series of bases are shown by closed circles on each broken line. The closed circles are on a straight line given by

$$
-\Delta H_{0}\left(\mathrm{kcal} \mathrm{mol}^{-1}\right)=0.013 \Delta v+0.91
$$

The $\Delta H_{0}$ of the PES/Phenoxy mixture estimated by eq 11 is $-1.95 \mathrm{kcal} \mathrm{mol}^{-1}$.

Since the enthalpy of mixing $\Delta H_{\text {mix }}$ is described by $\Delta H_{\text {mix }}=\psi \Delta H_{0}, \chi_{\mathrm{H}}$ is given by 


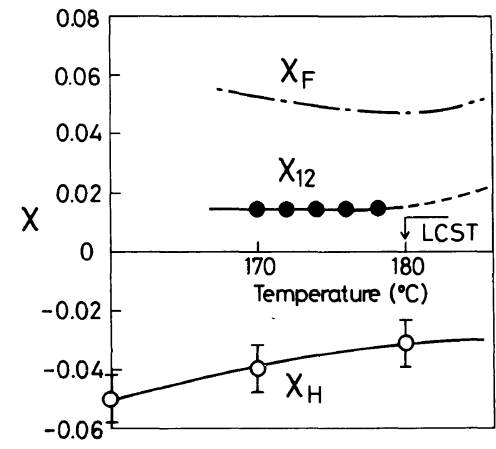

Figure 8. Temperature dependence of $\chi_{12}$ and its components. $\chi_{12}$ was determined by light scattering (below LCST) and the curve fitting to phase diagram (above LCST) while, $\chi_{\mathrm{H}}$, by FT-IR, and $\chi_{\mathrm{F}}=\chi_{12}-\chi_{\mathrm{H}}$.

$$
\chi_{\mathrm{H}}=\frac{\psi \Delta H_{0}}{R T \phi_{1} \phi_{2}}
$$

The $\chi_{\mathrm{H}}$ obtained by eq 11 and 12 is shown as a function of temperature in Figure 8.

\section{Temperature Dependence of $\chi_{\mathrm{F}}$}

In Figure 8 is also shown the $\chi_{12}(T)$ curve obtained by combining the results of the light scattering experiment below LCST and those by curve fitting of the phase diagram above LCST. According to recent understanding of polymer-polymer miscibility, ${ }^{1} \chi_{\mathrm{F}}$ may be given by the difference between $\chi_{12}$ and $\chi_{\mathrm{H}}\left(\chi_{12}=\right.$ $\left.\chi_{\mathrm{H}}+\chi_{\mathrm{F}}\right)$. The $\chi_{\mathrm{F}}(T)$ thus obtained is shown by the chain line in Figure 8. It is concave with a minimum. In other words, the free volume term becomes bigger with increasing temperature, as predicted theoretically, and also becomes bigger with decreasing temperature at the low temperature region. Such temperature dependence may be interpreted by the modified version of the equation-of-state theory. ${ }^{20}$ The concave nature in $\chi_{\mathrm{F}}(T)$ is empirically justified, providing a deeper understanding of the thermodynamics of polymer-polymer mixture. It leads to an interpretation of the appearance of both LCST and UCST in the mixtures of dissimilar polymers with high molecular weights. ${ }^{2-4}$

\section{CONCLUDING REMARKS}

The concave nature in $\chi_{\mathrm{F}}(T)$ is suggested in our PES/Phenoxy system. The physical meaning of $\chi_{\mathrm{F}}(T)$ should be given by the thermodynamic interpretation in tems of the structure in a single phase mixture. We believe that the short-range ordering in miscible polymer blend, proved by depolarized light scattering, ${ }^{21}$ may provide key evidence for this feature.

\section{REFERENCES AND NOTES}

1. D. R. Paul and S. Newman Ed., "Polymer Blends," Academic Press, New York, N.Y., 1978.

2. T. Ougizawa, T. Inoue, and H. W. Kammer, Macromolecules, 18, 2089 (1985).

3. G. Cong, Y. Haung, W. J. Macknight, and F. E. Karasz, Macromolecules, 19, 2765 (1986).

4. H. Saito, Y. Fujita, and T. Inoue, Polym. J., 19, 405 (1987).

5. F. S. Bates, G. D. Wignall, and W. C. Koehler, Phys. Rev. Lett., 55, 2425 (1985).

6. T. Ougizawa and T. Inoue, Polym. J., 18, 521 (1986).

7. B. S. Morra and R. S. Stein, J. Polym. Sci., Polym. Phys. Ed., 20, 2243 (1982).

8. J. E. Harris, D. R. Paul, and J. W. Barlow, Polym. Eng. Sci., 23, 676 (1983).

9. J. H. Wendorff, J. Polym. Sci., Polym. Lett. Ed., 18, 439 (1980).

10. M. Shibayama, H. Yang, R. S. Stein, and C. C. Han, Macromolecules, 18, 2179 (1985).

11. P. G. de Gennes, "Scaling Concepts in Polymer Physics," Cornell University Press, New York, N.Y., 1979.

12. R. S. Drago, N. O'Bryan, and G. C. Vogel, J. Am. Chem. Soc., 92, 3924 (1970).

13. F. M. Fowkes, D. O. Tishler, J. A. Wolfe, L. A. Lannigan, C. M. Adamu-gohn, and M. J. Halliwell, J. Polym. Sci., Polym. Chem. Ed., 22, 547 (1984).

14. T. K. Kwei, E. M. Pearce, F. Ren, and J. P. Chen, J. Polym. Sci., Polym. Phys. Ed., 24, 1597 (1986).

15. V. B. Singh and D. J. Walsh, J. Macromol. Sci., Phys., B25, 65 (1986).

16. M. M. Coleman, P. C. Painter, Appl. Spect. Rev., 20, 255 (1984).

17. R. S. Stein and J. J. Keane, J. Polym. Sci., 17, 21 (1955).

18. J. Maruta, T. Ougizawa, and T. Inoue, Polymer, 29, 2056 (1988).

19. The spectrum of neat Phenoxy was also resolved into the three Lorentzians. A small band at intermediate region may be processed by the curve fitting with 
symmetric Lorentzians. The area of intermediate band of neat Phenoxy was subtracted from the $A_{\mathrm{HB}}$ of blend. After this correction, $\psi$ values were calculated. The $\psi$ value can be also estimated as the fraction of area of intermediate peak of the difference spectrum obtained by subtraction of the spectrum of neat Phenoxy from that of the blend. The $\psi$ thus obtained was equal to that by the peak resolution.

20. H. W. Kammer, T. Ougizawa, and T. Inoue, Polymer, 30, 888 (1989).

21. H. Saito, M. Matsuura, T. Okada, and T. Inoue, Polym. J., 21, 357 (1989). 